\title{
Effect on Ischemia of Nicardipine Hydrochloride in Acute Intracerebral Hemorrhage: Perfusion Computed Tomography Study
}

\author{
Do Kyeun Jeong, Sung-Kyun Hwang
}

Department of Neurosurgery, Mokdong Hospital, Ewha Womans University College of Medicine, Seoul, Republic of Korea

Corresponding author:

Sung-Kyun Hwang

Department of Neurosurgery,

Mokdong Hospital, Ewha Womans

University College of Medicine,

1071, Anyangcheon-ro,

Yangcheon-gu, Seoul 07985,

Republic of Korea

Tel: $+82-2-2650-2872$

Fax: $+82-2-2650-2652$

E-mail: nshsg@ewha.ac.kr

Received: September 4, 2018

Revised: September 10, 2018

Accepted: September 12, 2018
Objective: We performed brain perfusion computed tomography (CT) studies to correlate the effect of intravenous nicardipine hydrochloride $(\mathrm{HCl})$ with ischemia around the hematoma in patients with intracerebral hemorrhage (ICH). Methods: Thirty-two patients (mean age \pm range, $60 \pm 11.5$ years) with hypertensive ICH who visited a single center from March 2013 to December 2015 were treated with intravenous injection of nicardipine $\mathrm{HCl}$. Cerebral blood flow (CBF), maximum oxygen extraction fraction (OEFmax) and maximum cerebral metabolic rate of oxygen $\left(\mathrm{CMRO}_{2} \mathrm{max}\right)$ were measured by the brain perfusion $\mathrm{CT}$ to determine if there is any ischemia around the hematoma and the contralateral normal site at the time of admission and after $24 \mathrm{hr}$ twice. Results: Perihematoma data were compared with mirror contralateral normal region. Systolic blood pressure (BP) and diastolic BP were significantly decreased after nicardipine administration, and perihematoma CBF was not significant different from that of the normal contral lateral site after $24 \mathrm{hr}$ from admission. Mean OEFmax slightly was higher in the perilesional area than in the contralateral normal area and $\mathrm{CMRO}_{2}$ max of perilesional area was slightly lower than that of the contralateral area. But there were no significant changes between the two groups. Conclusion: This study suggests that lowering BP using nicardipine after ICH does not show significant changes in $\mathrm{CBF}$, OEFmax, and $\mathrm{CMRO}_{2}$ max measured by perfusion brain $\mathrm{CT}$. Based on this, it is possible to support the controlled use of intravenous nicardipine $\mathrm{HCl}$ at acute phase of hypertensive ICH. Large-scale controlled studies, long-term results are needed to evaluate the actual effect of the ischemic changes around the hematoma when using this drug to lower BP.

Key Words: Cerebral hemorrhage; Cerebrovascular circulation; Nicardipine; Perfusion imaging; Tomography, X-ray computed

\section{INTRODUCTION}

Spontaneous intracerebral hemorrhage (ICH) is one of the most serious complications that occur in patients with hypertension and atherosclerotic changes in blood vessels. Patients who diagnosed as ICH have a marked increase in blood pressure (BP) at the time of admission. Sustained increased BP in patients with ICH may lead to enlargement of the hematoma, so lowering $\mathrm{BP}$ is generally recommended in patients with acute $\mathrm{ICH}^{2,7,9,15,16)}$.

However, there are studies that refute these treatments because excess lowering BP after onset of ICH can lead to cerebral ischemia as a result of lowering cerebral blood flow (CBF). Other studies have suggested that localized ischemia and acidosis in the ICH and surrounding areas may impair the intrinsic brain protective mechanism due to compression of the micro- vasculature, resulting in regional reductions in $\mathrm{CBF}$ and ischemia at the distant area of $\mathrm{ICH}$. And, elevated $\mathrm{BP}$ can be a posi- tive result of Cushing-Kocher reaction to keep cerebral perfusion, and if it is lowered, it could be an adverse role that interferes with the protective mechanisms of blood vessels itself $3,5,6,8-10,17,18,21)$.

The imaging technique of perfusion computed tomography (CT) is useful to identify tissue hypoperfusion of surrounding the hematoma (penumbra), even though there was conflicting interpretation of ICH patients ${ }^{1,4,7,11,12,19,22)}$.

Therefore, we investigated the effect of BP on perihematoma ischemia to provide a reasonable basis for nicardipine hydrochloride $(\mathrm{HCl})$ therapy using a standardized $\mathrm{BP}$ management protocol in hypertensive ICH patients through this perfusion CT study. 


\section{MATERIALS AND METHODS}

This study was performed in 32 patients in our single center, and the same protocol was applied as a standard practice in all practices. This protocol was introduced as a standard practice for review by the Institutional Review Board. During the study period, all clinical data were collected for acute ICH patients treated with nicardipine $\mathrm{HCl}$ protocol.

\section{Patient Selection}

The 32 patients included in this study were those who visited our single institution between January 2013 and December 2015 and were treated within $6 \mathrm{hr}$ after the onset of symptoms. When the onset time was not described, the last time that was normal was regarded as the onset time. This study represents a continuous cohort.

Patient inclusion criteria were (1) no surgical hematoma removal was performed; (2) the patient with Glasgow coma scale is above 8 ; (3) The size of the hematoma measured by the formula of length $\times$ width $\times$ height $/ 2$ on CT is less than $60 \mathrm{~mL}^{11)}$; (4) Patients with hypertension with systolic BP $>140 \mathrm{mmHg}$ at admission. Patients exclusion criteria were: (1) If they can not reliably assess the onset time or have a symptom before $24 \mathrm{hr}$; (2) fother intracranial lesions such as an aneurysm, arteriovenous malformation, or tumor; (3) hematoma in the cerebellum, brainstem or only in ventricles; (4) when taking medication that promotes a history of coagulopathy or congenital coagulopathy status.

And we collected information about the following risk factors: age, sex, previous diagnosed hypertension, diabetes, previous cerebrovascular attack, chronic kidney disease, any heart disease in each patient. This information was collected based on information from patients or family members or from previous medical records.

\section{Treatment of Acute Hypertension Protocol}

The treatment group's BP target was to maintain systolic $\mathrm{BP}<140 \mathrm{mmHg}$ and diastolic BP $<100 \mathrm{mmHg}$ within $24 \mathrm{hr}$ of symptom onset. The initial treatment is as follows: nicardipine $\mathrm{HCl}$ was started intravenously at $10 \mathrm{mg} / \mathrm{hr}$ and increased to the maximum acceptable dose $(15 \mathrm{mg} / \mathrm{hr})$ by increasing 2.5 $\mathrm{mg} / \mathrm{hr}$ every $15 \mathrm{~min}$ if target BP was not achieved. BP was monitored at the physician's discretion using an intra-arterial catheter or non-invasive $\mathrm{BP}$ monitor. If the systolic BP drops under $110 \mathrm{mmHg}$, or diastolic BP drops under $70 \mathrm{mmHg}$, or if any symptoms due to hypotension are present, the antihypertensive treatment is discontinued and additional care is performed immediately by the physician.

\section{Imaging Protocol}

All patients underwent non-contrast CT immediately after visit to the emergency room for diagnosis.

If the patient was to be eligible, additional brain perfusion CT images were added, followed immediately by IV nicardipine, and brain perfusion CT images were taken again after $24 \mathrm{hr}$ to discriminate the change of $\mathrm{CBF}$ on perihematoma region. The perfusion CT images were obtained by intravenous iodide contrast agent $(40 \mathrm{~mL})$ for more than $10 \mathrm{sec}$ and CT images were collected for $50 \mathrm{sec}(80 \mathrm{kVp}, 200 \mathrm{~mA}$ per image) every 1 sec.

\section{Measurement of Variables}

Raw contrast-enhanced CT images were transferred to a personal computer workstation and analyzed using a custom designed software package. Manual selection of a contralateral site of hematoma was used for correction of bolus delay and dispersion with a singular value of deconvolution algorithm. Quantitative perfusion maps including CBF were generated on a voxel-wise basis. From voxel-wise tissue impulse response curves, maximum oxygen extraction fraction (OEFmax) maps was designed based on transit time parameters. Arterial concentration of oxygen $\left(\mathrm{CaO}_{2}\right)$ was estimated from arterial blood gas analysis from individual patients and the maximum cerebral metabolic rate of oxygen $\left(\mathrm{CMRO}_{2} \mathrm{max}\right)$ was derived from $\mathrm{CBF}$ $\times$ OEFmax $\times \mathrm{CaO}_{2}$ on a voxel-wise basis (Fig. 1).

\section{Statistical Analysis}

Statistical analysis was performed with t-test of linear mixed mode, Wilcox rank sum test and Fisher exact test. A p-value of 0.05 or less was considered to be statistically significant.
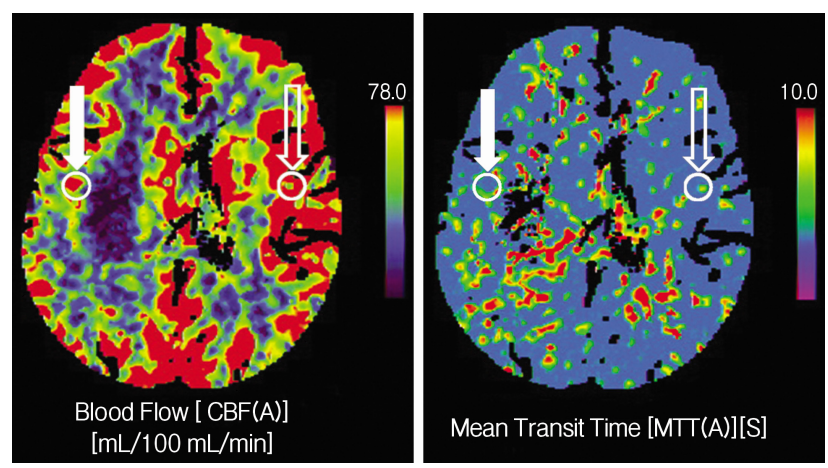

Fig. 1. A 36-year-old woman with right putaminal hemorrhage. (A) Region of interests (ROls) are drown in both hemispheres of cerebral blood flow map (empty circles). In right hemisphere, affected ROI is placed around the hematoma (filled arrow), and contralateral normal $\mathrm{ROI}$ is placed in same site of normal hemisphere (empty arrow). (B) Mean transit time is also measured at same ROls of both hemispheres. 
Continuous variables were expressed as mean values and categorical variables were expressed as frequency.

\section{RESULTS}

A total of 32 patients were studied, 18 were men (56.3\%) and $14(43.8 \%)$ were women and their average age was 60 years (range, 25-84 years). Among the all subjects, 27 patients had a history of hypertension, a risk factor for ICH, was already diagnosed and the patient was taking medication. Other risk factor was diabetes (3), coronary heart disease (2), stroke (5), and end stage renal disease (1). Patients with coagulopathy, including platelet function, were excluded. On admission, the mean Glasgow coma scale was $12.9 \pm 1.1$. ICH was mainly observed in typical locations (putamen 16, thalamus 13, lobar 3 ) and, in most cases, small-to-medium in volume (mean hemorrhage volume 19.3 \pm 11.6 ) (Table 1).

During the first $24 \mathrm{hr}$ after admission, the BP of all patients was recorded hourly. Initial mean systolic and diastolic BPs were $176.8 \pm 15.6 \mathrm{mmHg}$ and $94 \pm 13.9 \mathrm{mmHg}$, respectively.

Administration of nicardipine $\mathrm{HCl}$ by protocol reduced mean BP for the first $2 \mathrm{hr}$ immediately after infusion, but did not show any decrease in BP until $24 \mathrm{hr}$. Mean systolic and diastolic BPs for $24 \mathrm{hr}$ were $136.6 \pm 17.8 \mathrm{mmHg}$ and $74.5 \pm 12.5 \mathrm{mmHg}$

Table 1. Baseline characteristics of study patients $(n=32)$

\begin{tabular}{lc}
\hline \hline & Mean \pm range or $\mathrm{n}(\%)$ \\
\hline Mex & $18(56.3)$ \\
Female & $14(43.8)$ \\
Age & $60.0 \pm 11.5$ \\
Glasgow Coma Scale & $12.9 \pm 1.1$ \\
Location & \\
Putamen & $16(50.0)$ \\
Thalamus & $13(40.6)$ \\
Lobar & $3(9.4)$ \\
Intraventricular hemorrhage & $3(9.4)$ \\
Hematoma size & $19.3 \pm 11.6$ \\
Overall volume & $28(87.5)$ \\
Small (<30 cc) & $4(12.5)$ \\
Medium (30-60 cc) & \\
Risk factors & $27(84.4)$ \\
Hypertension & $3(9.4)$ \\
Diabetes mellitus & $2(6.3)$ \\
Coronary disease & $5(15.6)$ \\
CVA & $1(3.1)$ \\
End-stage renal disease &
\end{tabular}

The data is presented as mean \pm range or number (\%). CVA: cerebrovascular attack. after injection, respectively ( $\mathrm{p}<0.001$, mixed effect linear model).

Initial CBF of affected perihematoma region was (51 \pm 8.3 / $100 \mathrm{~g} / \mathrm{min}$ ), and ipsilateral normal tissue CBF was slightly increased $(43 \pm 12.8 / 100 \mathrm{~g} / \mathrm{min})$ and $\mathrm{p}$-value was 0.32 . After 24 $\mathrm{hr}$ continuous intravenous infusion of nicardipine $\mathrm{HCl}, \mathrm{CBF}$ in normal tissues was slightly increased $(46 \pm 9.5 / 100 \mathrm{~g} / \mathrm{min})$ compared to the that of admission, whereas affected CBF was slightly decreased (49 $\pm 10.2 / 100 \mathrm{~g} / \mathrm{min})$, but $\mathrm{p}$ value did not show significant statistical difference.

In addition, the affected OEFmax $(0.44 \pm 0.18)$ and the normal region $(0.45 \pm 0.23)$ were almost the same at first, but after 24 $\mathrm{hr}$, the affected OEFmax $(0.43 \pm 0.36)$ showed slightly decreased data compared to the normal region $(0.48 \pm 0.08)$ But did not show any statistically significant differences $(\mathrm{p}=0.71)$ Although the absolute value of OEFmax was slightly reduced, the oxygen supply to the tissue was within the normal range. The value of $\mathrm{CMRO}_{2}$ max around the hematoma was also not different between the initial normal tissue and the affected tissue $(3.29 \pm 1.55 \mathrm{~mL} / 100 \mathrm{~g} / \mathrm{min}, 3.15 \pm 1.27 \mathrm{~mL} / 100 \mathrm{~g} / \mathrm{min} ; \mathrm{p}=$ $0.76)$, and there was no significant difference between the two tissues after $24 \mathrm{hr}(3.19 \pm 1.36 \mathrm{~mL} / 100 \mathrm{~g} / \mathrm{min}$ vs. $3.28 \pm 2.08 \mathrm{~mL} /$ $100 \mathrm{~g} / \mathrm{min} ; \mathrm{p}=0.82$ ) (Table 2).

\section{DISCUSSION}

Treatment of acute hypertension in patients with hypertensive ICH - to what extent it is necessary to lower BP is still controversial. The persistent severe acute hypertension (>200 mm/Hg) may cause abrupt elevation of cerebral perfusion pressure (CPP), which may increase the size of the existing hematoma and exacerbate the edema, intracranial pressure

Table 2. Results of blood pressure, cerebral blood flow, maximum oxygen extraction fraction, and maximum cerebral metabolic rate of oxygen

\begin{tabular}{lcc}
\hline \hline Parameter & Baseline & $24 \mathrm{hr}$ \\
\hline Mean SBP $(\mathrm{mmHg})$ & $176.8 \pm 15.6$ & $136.6 \pm 17.8$ \\
Mean DBP $(\mathrm{mmHg})$ & $94.0 \pm 13.9$ & $74.5 \pm 12.5$ \\
$\mathrm{CBF}$ normal $(\mathrm{mL} / 100 \mathrm{~g} / \mathrm{min})$ & $43.0 \pm 12.8$ & $46.0 \pm 9.5$ \\
$\mathrm{CBF}$ affected $(\mathrm{mL} / 100 \mathrm{~g} / \mathrm{min})$ & $51.0 \pm 8.3$ & $49.0 \pm 10.2$ \\
p-value & 0.32 & 0.29 \\
OEFmax normal & $0.45 \pm 0.23$ & $0.48 \pm 0.08$ \\
OEFmax affected & $0.44 \pm 0.18$ & $0.43 \pm 0.36$ \\
p-value & 0.83 & 0.71 \\
$\mathrm{CMRO}_{2}$ normal $(\mathrm{mL} / 100 \mathrm{~g} / \mathrm{min})$ & $3.29 \pm 1.55$ & $3.28 \pm 2.08$ \\
$\mathrm{CMRO}_{2}$ affected $(\mathrm{mL} / 100 \mathrm{~g} / \mathrm{min})$ & $3.15 \pm 1.27$ & $3.19 \pm 1.36$ \\
p-value $^{*}$ & 0.76 & 0.82 \\
\hline $\mathrm{SBP}^{*} \mathrm{sys}^{*}$ & &
\end{tabular}

SBP: systolic blood pressure; DBP: diastolic blood pressure; CBF: cerebral blood flow; OEFmax: maximum oxygen extraction fraction; $\mathrm{CMRO}_{2}$ : cerebral metabolic rate of oxygen. "p-value $<0.05$ : statistically significant. 
(ICP) leading to cerebral ischemia.

However, on the other hand, a rapid drop in BP that causes loss of autoregulatory mechanisms around the hematoma can reduce $\mathrm{CBF}$ in and around the $\mathrm{ICH}$, resulting in ischemia and cerebral perfusion failure ${ }^{5,6,8,10,17,21)}$. Due to the ability of the autoregulation to maintain $\mathrm{CBF}$ as a change in diameter of the arteriole relative to changes in $\mathrm{CPP}$, changes in $\mathrm{BP}$ within the normal range have little or no effect on $\mathrm{CBF}$.

Under normal circumstances, the relationship between ICP and CPP can be maintained with autoregulatory mechanism. $\mathrm{CBF}$ will return to normal range within a few sec because there is vasodilatation of cerebral resistance vessels, decrease in cerebrovascular resistance and increase in cerebral blood volume as the CPP falls within the autoregulation range. However, if this condition persists, the autoregulation ability will not work and eventually causes a decrease in CBF, resulting in ischemia ${ }^{3,5,6,8-10,17,18,21)}$.

Previous studies have demonstrated an early phase of reduction process of $\mathrm{CBF}$ around $\mathrm{ICH}$. The initial decrease in CBF immediately after ICH appears to reflect the immediate and hemodynamic reaction, and a raised ICP was observed as a compensatory mechanism that makes $\mathrm{CBF}$ normal1 $1^{3,20,21)}$. Nath et al. ${ }^{13)}$ showed 10 min of ischemia immediately after ICH and showed recovery of $\mathrm{CBF}$ to normal within $3 \mathrm{hr}$. Similarly, Yang et $\mathrm{al}^{20)}$ confirmed that CBF with ICH reduced by $50 \%$ returned to normal after $4 \mathrm{hr}$.

Powers et all. ${ }^{14)}$ reported that 14 ICH patients with nicardipine infusion using a positron emission tomographic scan showed no effect on CBF of perihematoma area and ipsilateral normal region, while decrease in mean arterial pressure about $17 \%$ from baseline. In general, previous studies suggest that changes in CBF after a decrease in mean BP are not consistent with ICH because self-regulation is maintained after $\mathrm{ICH}^{1,5,13,21)}$. We have not found any negative effects of reducing mean perfusion over a 24-hr period and no evidence of ischemia was found in any patient. The results of our study are consistent with previous studies mentioned above. Decreased cerebral vascular resistance by nicardipine causes compensatory vasodilation, suggesting that $\mathrm{CBF}$ is maintained. This suggests that the controlled use of nicardipine $\mathrm{HCl}$ after ICH significantly reduces the mean $\mathrm{BP}$, meaning that $\mathrm{CBF}, \mathrm{OEF}, \mathrm{CMRO}_{2}$ is maintained simultaneously.

There are some limitations in our studies. First, patients with a hematoma volume less than $60 \mathrm{~mL}$ and patients with good Glasgow Coma Scale were selected. In patients with larger amount of ICH, a higher ICP is induced, which requires a rapid increase in systemic BP and a sudden change in autoregulation. Relatively, patients with smaller hematoma investigated in our study have lesser changes in ICP, so the effect of nicardipine on changes in $\mathrm{CBF}$ may be compensable, but it is difficult to assume that blood flow is still maintained even in large hematomas because we did not measure the ICP of patients directly. Second, this study of a relatively small number of patients (32 patients) is not sufficient to conclude definitively. Third, the existence of unknown, confusing variables not explained in the final analysis can not be ruled out. The possibility that dynamic cerebral autoregulation has already been impaired by an already existing vascular risk factor such as chronic hypertension, diabetes and silent infarction can not be ruled out.

\section{CONCLUSION}

This study supports the controlled use of nicardipine $\mathrm{HCl}$ for acute phase ICH based on no significant changes in perfusion around the ICH with a meaningful decrease in mean BP. If the reduction in $\mathrm{BP}$ affects $\mathrm{CBF}$, OEF, and $\mathrm{CMRO}_{2}$, requiring more research based on large, well-designed, randomized, controlled, and long-term outcomes. Our study can help to design such trials and can provide useful guidance when lowering $\mathrm{BP}$ is needed in patients with $\mathrm{ICH}$.

\section{CONFLICT OF INTEREST}

No potential conflict of interest relevant to this article was reported.

\section{REFERENCES}

1. Beseoglu K, Etminan N, Turowski B, Steiger HJ, Hanggi D: The extent of the perihemorrhagic perfusion zone correlates with hematoma volume in patients with lobar intracerebral hemorrhage. Neuroradiology 56:535-541, 2014

2. Diedler J, Sykora M, Rupp A, Poli S, Karpel-Massler G, Sakowitz O, et al.: Impaired cerebral vasomotor activity in spontaneous intracerebral hemorrhage. Stroke 40:815-819, 2009

3. Etminan N, Beseoglu K, Turowski B, Steiger HJ, Hänggi D: Perfusion CT in patients with spontaneous lobar intracerebral hemorrhage: effect of surgery on perihemorrhagic perfusion. Stroke 43:759-763, 2012

4. Fainardi E, Borrelli M, Saletti A, Schivalocchi R, Azzini C, Cavallo $\mathrm{M}$, et al.: CT perfusion mapping of hemodynamic disturbances associated to acute spontaneous intracerebral hemorrhage. Neuroradiology 50:729-740, 2008

5. Gould B, McCourt R, Asdaghi N, Dowlatshahi D, Jeerakathil T, Kate M, et al.: Autoregulation of cerebral blood flow is preserved in primary intracerebral hemorrhage. Stroke 44:17261728, 2013

6. Gould B, McCourt R, Gioia LC, Kate M, Hill MD, Asdaghi $\mathrm{N}$, et al.: Acute blood pressure reduction in patients with intracerebral hemorrhage does not result in borderzone region hypoperfusion. Stroke 45:2894-2899, 2014

7. Herweh C, Jüttler E, Schellinger PD, Klotz E, Jenetzky E, Orakcioglu $\mathrm{B}$, et al.: Evidence against a perihemorrhagic penumbra 
provided by perfusion computed tomography. Stroke 38:29412947, 2007

8. Hwang SK, Kim JS, Kim JH, Hong CK, Yang KH: Antihypertensive treatment of acute intracerebral hemorrhage by intravenous nicardipine hydrochloride: prospective multi-center study. J Korean Med Sci 27:1085-1090, 2012

9. Kate MP, Hansen MB, Mouridsen K, Østergaard L, Choi V, Gould BE, et al.: Blood pressure reduction does not reduce perihematoma oxygenation: a CT perfusion study. J Cereb Blood Flow Metab 34:81-86, 2014

10. Klingelhfer J, Sander D: Doppler CO2 test as an indicator of cerebral vasoreactivity and prognosis in severe intracranial hemorrhages. Stroke 23:962-966, 1992

11. Kothari RU, Brott T, Broderick JP, Barsan WG, Sauerbeck LR, Zuccarello M, et al.: The ABCs of measuring intracerebral hemorrhage volumes. Stroke 27:1304-1305, 1996

12. Kuwata N, Kuroda K, Funayama M, Sato N, Kubo N, Ogawa A: Dysautoregulation in patients with hypertensive intracerebral hemorrhage. A SPECT study. Neurosurg Rev 18:237-245, 1995

13. Nath FP, Kelly PT, Jenkins A, Mendelow AD, Graham DI, Teasdale GM: Effects of experimental intracerebral hemorrhage on blood flow, capillary permeability, and histochemistry. J Neurosurg 66:555-562, 1987

14. Powers WJ, Zazulia AR, Videen TO, Adams RE, Yundt KD, Aiyagari V, et al.: Autoregulation of cerebral blood flow surrounding acute (6 to 22 hours) intracerebral hemorrhage. Neurology 57:18-24, 2001

15. Qureshi AI, Mohammad YM, Yahia AM, Suarez JI, Siddiqui AM, Kirmani JF, et al.: A prospective multicenter study to evaluate the feasibility and safety of aggressive antihypertensive treatment in patients with acute intracerebral hemorrhage. J Intensive Care Med 20:34-42, 2005

16. Qureshi AI, Tuhrim S, Broderick JP, Batjer HH, Hondo H, Hanley DF: Spontaneous intracerebral hemorrhage. N Engl J Med 344:1450-1460, 2001

17. Reinhard M, Neunhoeffer F, Gerds TA, Niesen WD, Buttler KJ, Timmer J, et al.: Secondary decline of cerebral autoregulation is associated with worse outcome after intracerebral hemorrhage. Intensive Care Med 36:264-271, 2010

18. Rosand J, Eskey C, Chang Y, Gonzalez RG, Greenberg SM, Koroshetz WJ: Dynamic single-section CT demonstrates reduced cerebral blood flow in acute intracerebral hemorrhage. Cerebrovasc Dis 14:214-220, 2002

19. Siddique MS, Fernandes HM, Arene NU, Wooldridge TD, Fenwick JD, Mendelow AD: Changes in cerebral blood flow as measured by HMPAO SPECT in patients following spontaneous intracerebral haemorrhage. Acta Neurochir Suppl 76:517-520, 2000

20. Yang GY, Betz AL, Chenevert TL, Brunberg JA, Hoff JT: Experimental intracerebral hemorrhage: relationship between brain edema, blood flow, and blood-brain barrier permeability in rats. J Neurosurg 81:93-102, 1994

21. Zazulia AR, Diringer MN, Videen TO, Adams RE, Yundt K, Aiyagari V, et al.: Hypoperfusion without ischemia surrounding acute intracerebral hemorrhage. J Cereb Blood Flow Metab 21:804-810, 2001

22. Zhou J, Zhang H, Gao P, Lin Y, Li X: Assessment of perihematomal hypoperfusion injury in subacute and chronic intracerebral hemorrhage by CT perfusion imaging. Neurol Res 32: 642-649, 2010 